\section{Endoscopic full-thickness resection of a submucosal right colon lesion}

A novel endoscopic full-thickness resection device (Ovesco Endoscopy, Tübingen, Germany) has been developed based on the combination of an overthe-scope clip with an enlarged resection cap $(21 \mathrm{~mm})$ and a snare preloaded in the distal rim of the cap $[1,2]$. We report the case of a 53-year-old woman with a history of abdominal panniculitis with fat necrosis and perivasculitis with giant cell histiocytosis. She complained of weight loss, abdominal pain, and fever. Positron emission tomography (PET) scan showed fluorodeoxyglucose uptake

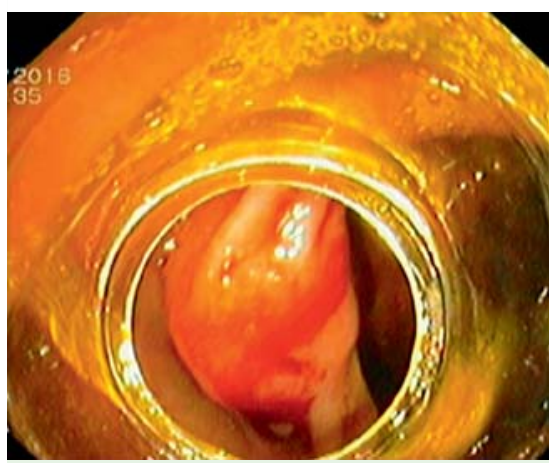

Fig. 1 View of the submucosal lesion in the right colon.

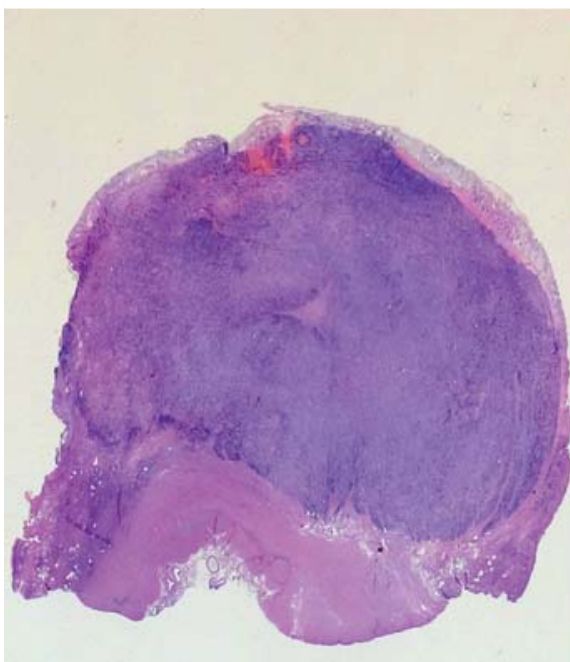

a

Fig. 4 Histological analysis of the lesion. a Whole-mount view of the histological slide of the entire excised specimen (hematoxylin and eosin [H\&E]). The muscularis propria layer is seen below and is stained pink. The lymphoid nodule is blue and is located entirely within the submucosa. The mucosa appears as a thin, clear, pinkish strip at the top. $\mathbf{b}$ Microscopic view of the histological section showing the mucosa in the top right side and part of the lymphoid proliferation in the lower left $(\mathrm{H} \& \mathrm{E}, \times 200)$. c CD20/CD3 double immunostained slide of the area shown in (b). Cells expressing CD20 are stained red and those expressing CD3 are stained brown. Most of the cells are seen to express CD20, and thus are of B-cell phenotype $(\times 400)$. doscopy) was ineffective. We therefore decided to capture the lesion using suction, and as soon as we were sure that the whole lesion was inside the cap, we deployed the preloaded clip. Resection was achieved using the integrated snare (Erbe ICC200, pure cut, 200W) ( Fig.2, - Fig. 3, $\bigcirc$ Video 1 ).

The procedure time was 21 minutes (including the time to reach the right colon), and conscious sedation with propofol was used. No antibiotic therapy was administered. No complications were reported and the patient was discharged the following day.

A definitive diagnosis of nodular submucosal localization of diffuse large B-cell lymphoma was made ( $\bullet$ Fig. 4).

This is a very unusual case of B-cell lymphoma because no lymphadenopathy

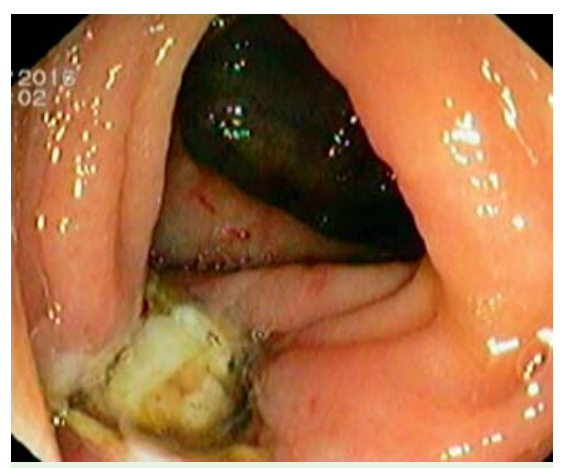

Fig. 2 View of the resection site: the clip sealed the colonic wall defect.

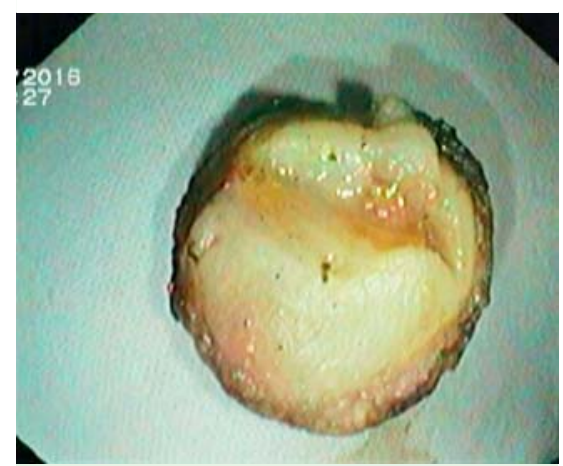

Fig. 3 The resected specimen.

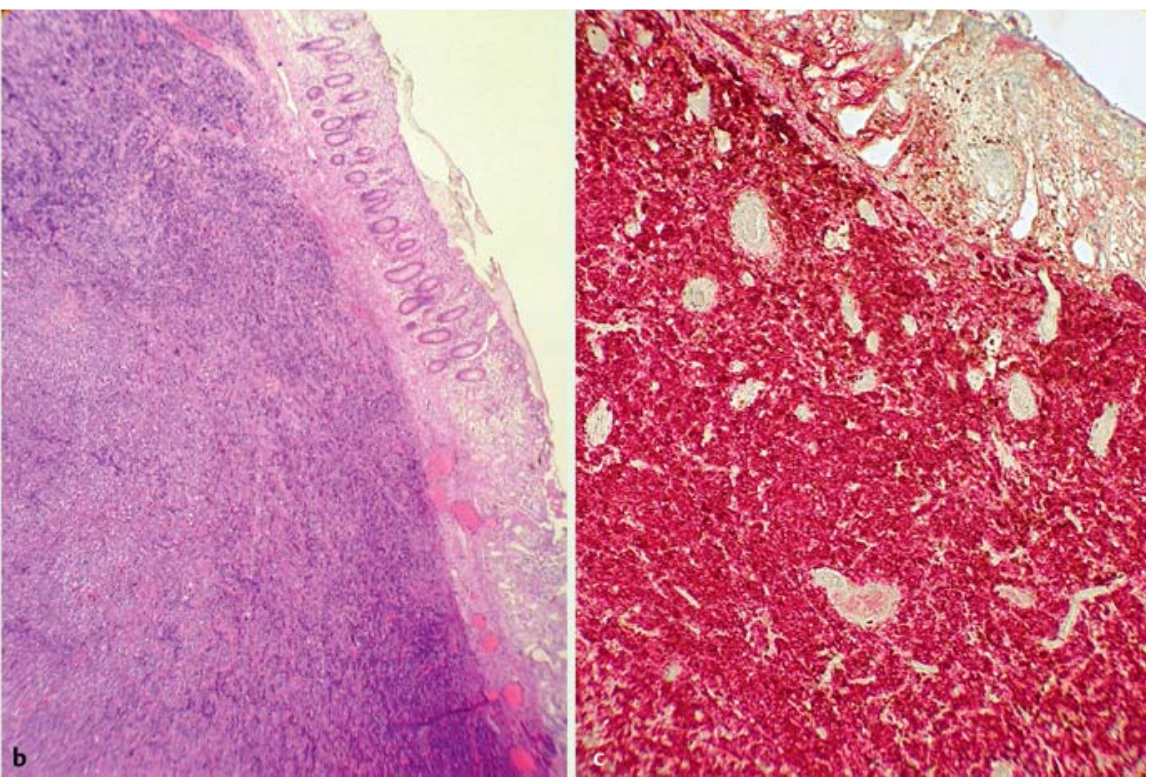

cap using the Twin grasper (Ovesco En- 


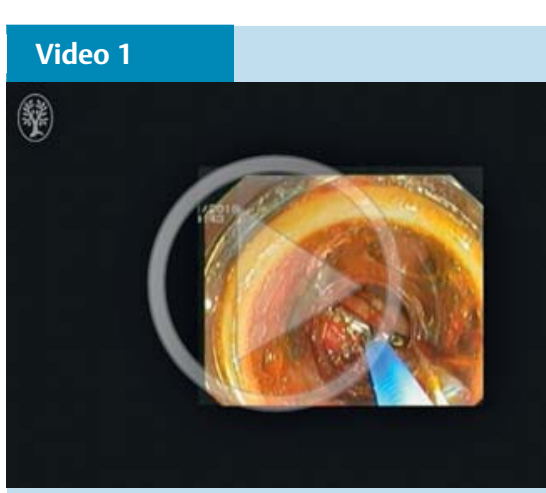

Use of a novel over-the-scope device to perform endoscopic full-thickness resection of a submucosal right colon lesion.

was detected at the PET and computed tomography scans; only a single $6-\mathrm{mm}$ hepatic nodule was identified. The patient is now receiving chemotherapy treatment with R-CHOP (rituximab, cyclophospha- mide, doxorubicin, vincristine, prednisolone).

This is the first case to be reported of EFTR of submucosal right colon lesion. Our experience shows the feasibility of this novel technique on submucosal lesions in the right colon. EFTR could represent a promising minimally invasive alternative to surgery in selected cases.

\section{Endoscopy_UCTN_Code_TTT_1AQ_2AD}

\section{Competing interests: None}

\section{Raffaele Salerno ${ }^{1}$, Giorgio Gherardi²,} Emanuela Paternò ${ }^{3}$, Sandro Ardizzone ${ }^{1}$

${ }^{1}$ Endoscopy Unit, ASST Fatebenefratelli Sacco, Milan, Italy

2 Department of Anatomic Pathology, ASST Fatebenefratelli Sacco, Milan, Italy

${ }^{3}$ Department of Oncology, ASST Fatebenefratelli Sacco, Milan, Italy

\section{References}

1 Schmidt A, Bauerfeind P, Gubler C et al. Endoscopic full-thickness resection in the colorectum with a novel over-the-scope device: first experience. Endoscopy 2015; 47: 719725

2 Fähndrich M, Sandmann M. Endoscopic fullthickness resection for gastrointestinal lesions using the over-the-scope clip system: a case series. Endoscopy 2015; 47: 76 - 79

3 Cai M, Zhou P, Lourenco LC et al. Endoscopic full-thickness resection (EFTR) for gastrointestinal subepithelial tumors. Gastrointest Endosc Clin N Am 2016; 26: 283-295

Bibliography

Dol http://dx.doi.org/

10.1055/s-0042-120338

Endoscopy 2016; 48: E376-E377

(c) Georg Thieme Verlag KG

Stuttgart · New York

ISSN 0013-726X

Corresponding author

Raffaele Salerno, MD

Endoscopy Unit

ASST Fatebenefratelli Sacco

Corso di Porta Nuova 23

Milan, 20121

Italy

Fax: +39-02-63632249

raffaele.salerno@asst-fbf-sacco.it 